\title{
Benchmarks
}

Holcombe Blvd., Houston, TX 77030, USA. e-mail:mwilkins@notes.mdacc.tmc.edu

Received 11 April 2000; accepted 11 August 2000.

\author{
Jun Wang and Miles F. \\ Wilkinson \\ The University of Texas M.D. \\ Anderson Cancer Center \\ Houston, TX, USA
}

\section{Construction of a cDNA Encoding a Repetitive Amino Acid Sequence}

BioTechniques 29:978-981 (November 2000)

PCR is a powerful method with which to generate almost any desired cDNA sequence. Construction of cDNA fragments that encode repetitive amino acid sequences by PCR has proven problematic, however, because repetitive nucleotide primers tend to amplify undesired fragments because of their mis-annealing with the template. To overcome this problem, Dom browski and Wright (2) developed a solid-phase gene assembly protocol, but this technique requires preparation of a solid-phase reaction system and thus cannot be routinely performed in most laboratories. Here, we describe a new strategy that makes use of standard PCR protocols, yet enables assembly of cDNAs encoding repetitive amino acid sequences.

The peptide unit Ala-Thr-Pro-AlaPro (ATPAP) is reported to be the shortest that can still function as an acceptor for $N$-acetylgalactosaminyltransferase-I (4). By introducing this peptide into a secretable form of fibroblast growth factor (FGF) (3) and expressing it in Chinese hamster ovary cells, we were able to produce FGF modified with a single $O$-glycan moiety (1). Subsequently, neo- $O$-glycosylated forms of FGF containing multiple $O$-glycans, similar to mucin-type glycoproteins, were created using two
cDNAs encoding FGFs with 10 repeats of the pentapeptide $O$-glycosylation unit at the $\mathrm{N}$ - or $\mathrm{C}$-terminus. In this report, the creation of the $\mathrm{N}$-terminal modified form is described in detail.

The amino acids comprising the pentapeptide $O$-glycosylation unit, alanine, proline and threonine, are encoded by codons GCX, CCX and ACX, respectively, where $\mathrm{X}$ is any nucleotide. We therefore designed four nucleotide cassettes that each encoded ATPAP and differed from one another at the last nucleotide for each codon (Figure 1A). To avoid mis-annealing the cassettes, they were linked together so that they encoded more than three repeats of the ATPAP unit (Figure 1B).

Generation of clones encoding the modified FGF entailed performing two rounds of PCR. The primers for the first PCR were 5'-TTCGAATTCCCAAGG CACGCTACTGGATGCTACTCCTGCTCCTGCGACGCCGGCGCCGGCA3'

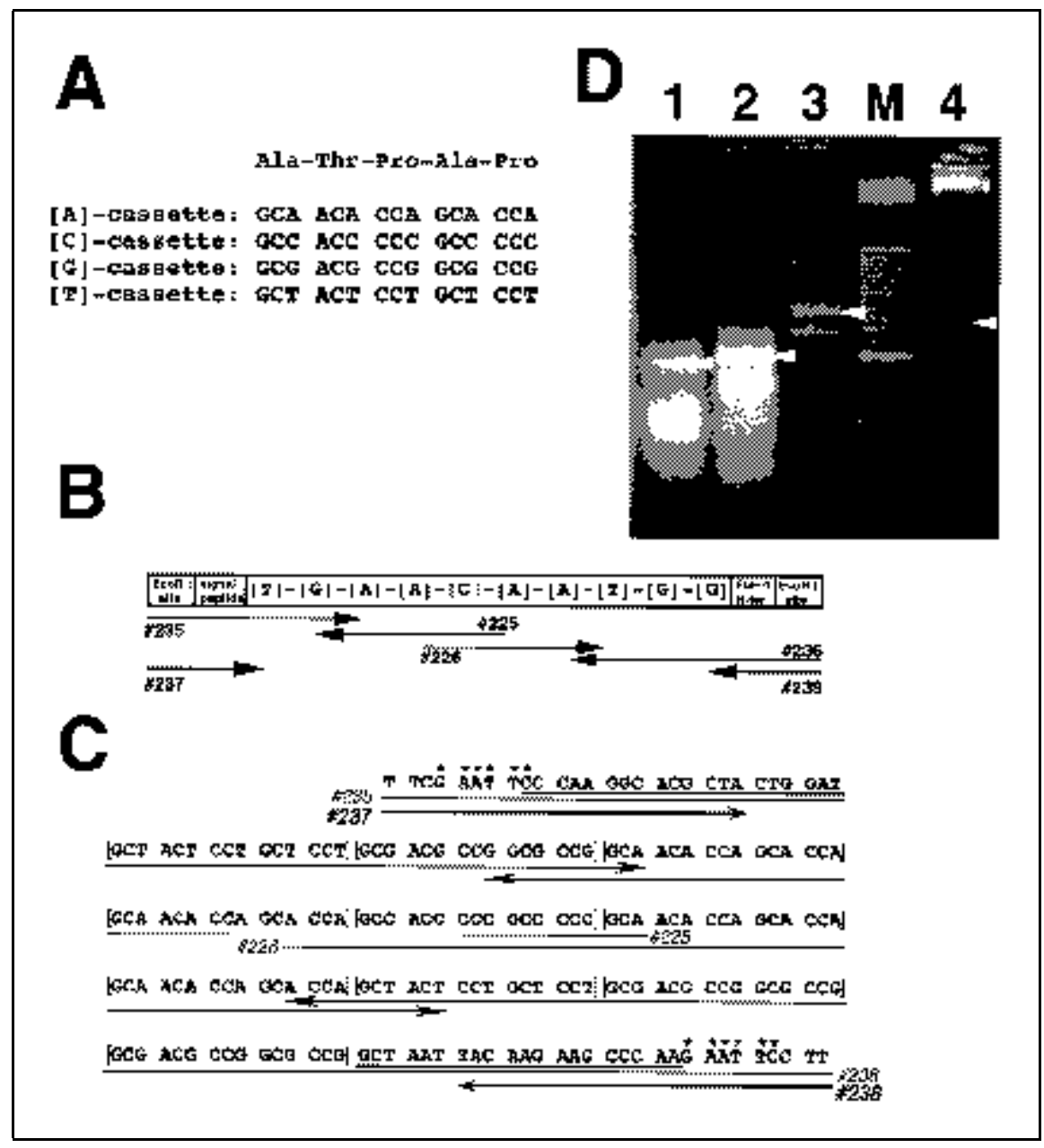

Figure 1. Construction of a cDNA encoding a repetitive amino acid sequence. (A) Four oligonucleotide cassettes, each encoding the pentapeptide $O$-glycosylation unit ATPAP. (B) Schematic diagram of the construction of a cDNA encoding the [ATPAP $]_{10}$ cassette, which is to be inserted between the signal peptide and the N-terminus of FGF-1. Each $O$-glycosylation unit is indicated by a bracket containing the symbol of the last nucleotide in each codon, as shown in panel A. PCR protocols were initially carried out separately using oligonucleotides \#235 and \#225 or \#226 and \#236 without a template. A mixture of the two reaction products was then subjected to PCR using primers \#237 and \#238. (C) Alignment of the primers with the completed sequence. The nucleotide sequences encoding the signal peptide and the FGF-1 N-terminus are underlined. The EcoRI recognition sites are indicated by asterisks. Primers are shown by arrows. (D) Agarose gel (4\%) electrophoresis of the PCR products and the enzyme digest of the final clone. Lane 1, the first PCR product obtained using \#235 and \#225 (106 bp); lane 2, the first PCR product obtained using \#226 and \#236 (123 bp); lane 3, the second PCR product (207 bp); lane M, 25-bp ladder markers (Life Technologies, Rockville, MD, USA); lane 4, EcoRI digest of the clone N$[\mathrm{ATPAP}]_{1} \sigma^{\mathrm{secFGF} / \mathrm{pBS} / \# 9}$ (195 bp). Arrowheads indicate the DNA bands having the expected sizes. 
(\#235); 5'-TGCGGGGGCGGGGGTG GCTGGTGCTGGTGTTGCTGGTGCTGGTGTTGCCGGCGCC-3' (\#225); 5 -ACCAGCCACCCCCGC CCCCGCA ACACCAGCACCA GCA AC ACCAGCACCAGCTACT-3' (\#226); and 5'-AAGGAATTCTTGGGCTTCTTGTAATAGCCGGCGCCGGCGTCGCCGGCGCCGGCGTCGCAGGAGCA GGAGTAGCTGGT-3' (\#236). Without template DNA, the two combinations of forward and reverse primers (\#235 and \#225, \#226 and \#236, 8 pmol/ $\mu \mathrm{L}$ each) were mixed individually with $\mathrm{dNTP}(0.2$ $\mathrm{mM}$ ) and subjected to PCR using Ta$\mathrm{KaRaTaq}^{\mathrm{TM}}$ DNA polymerase (TaKaRa Shuzo, Kyoto, Japan); the amplification protocol consisted of 30 cycles at $94^{\circ} \mathrm{C}$ for $30 \mathrm{~s}, 68^{\circ} \mathrm{C}$ for $30 \mathrm{~s}$ and $72^{\circ} \mathrm{C}$ for $30 \mathrm{~s}$. After agarose gel electrophoresis, the distinct bands at 106 and $123 \mathrm{bp}$ (Figure $1 \mathrm{D}$, lanes 1 and 2, respectively) were excised, and DNAs were extracted. The DNAs were mixed and used as the tem plate in a second PCR. The primers for this reaction were $5^{\prime}$-TTCGAATTCCCAAGGCACGCTA-3' (\#237) and 5'AAGGAATTCTTGGGCTTCTTGTA$3^{\prime}$ (\#238), and the amplification protocol was the same, except that annealing was performed at $60^{\circ} \mathrm{C}$. The reaction product was resolved by agarose gel electrophoresis, and a 207-bp DNA fragment (Figure $1 \mathrm{D}$, lane 3 , arrowhead) was isolated. After digestion with EcoRI (TaKaRa Shuzo), the fragment was subcloned into pBluescript ${ }^{\circledR} I I(K S+)$ vector (Stratagene, La Jolla, CA, USA) using a DNA Ligation Kit Ver. 2 (TaKaRa Shuzo), and the resultant ligation mixture was used to transform DH5 $\alpha$ E. coli cells.

Positive clones were initially selected according to their ampicillin resistance and white color. The clones were then screened by direct PCR using vector primers to reveal the size of the inserted sequence, and several with inserts of the expected size were obtained. The size of the harbored insert was further verified in one of the clones, designated N-[ATPAP $]_{10}$-secFGF/pBS/\#9, by digestion with EcoRI (Figure 1D, lane 4), and the correctness of its sequence was confirmed by direct sequencing (not shown). This DNA was further ligated with the signal sequence of FGF-6 at 5'-terminal and FGF-1 cDNA at $3^{\prime}$-terminal and constructed cDNA that encodes secretable FGF-1 carrying clustered mucin. By subcloning this cDNA into an expression vector and transfecting mammalian cells, we obtained a secreted FGF with a protein core of expected size and the correct N-terminal sequence (unpublished results). We were also able to use the same strategy to successfully introduce ATPAP repeat units at the C-terminus (unpublished results). This strategy should be effective for introducing ATPAP units into any protein of interest; moreover, it should also enable the creation of cDNAs encoding other repetitive short peptides.

\section{REFERENCES}

1.Asada, M., N. Orikasa, A. Yoneda, Y. Oda, K. Ota and T. Imamura. 1999. The AATPAP sequence is a very efficient signal for $O$-glycosylation in $\mathrm{CHO}$ cells. Glycoconj. J. 16:321-326.

2.Dombrowski, K.E. and S.E. Wright. 1992. Construction of a multiple mucin tandem repeat with a mutation in the tumor-specific epitope by a solid-phase gene assembly protocol. Nucleic Acids Res. 20:6743-6744.

3.Yoneda, A., M. Asada, M. Suzuki and T. Imamura. 1999. Introduction of an $\mathrm{N}$-glycosylation cassette into proteins at random sites: expression of neoglycosylated FGF. BioTechniques 27:576-582.

4. Yoshida, A., M. Suzuki, H. Ikenaga and M. Takeuchi. 1997. Discovery of the shortest sequence motif for high level mucin-type $O$-glycosylation. J. Biol. Chem. 272:16884-16888.

This study was supported in part by the Complexed Carbohydrate program of Agency of Industrial Science and Technology (AIST) to T.I. and M.A. A.Y. was supported by New Energy and Technology Development Organization (NEDO) fellowship. Address correspondence to Dr. Toru Imamura, Biosignaling Department, National Institute of Bioscience and Human Technology, Agency of Industrial Science and Technology 1-1 Higashi, Tsukuba, Ibaraki 3058566, Japan.e-mail: imamura@nibh.go.jp

Received 3 May 2000; accepted 20 July 2000.

Masahiro Asada, Atsuko
Yoneda, Yuko Oda and
Toru Imamura
National Institute of Bioscience
and Human Technology
Tsukuba, Ibaraki, Japan

Masahiro Asada, Atsuko Yoneda, Yuko Oda and Toru Imamura National Institute of Bioscience Tsukuba, Ibaraki, Japan 